\title{
Determinación de la competitividad en países de América Latina: aplicación de un nuevo método
}

\author{
Lourdes Gabriela Daza Aramayo*
}

\section{Resumen}

En este trabajo se calculó un índice de competitividad para diecisiete países de América Latina que denota la posición de cada uno de ellos, considerando la comparación de ocho indicadores en cuatro dimensiones y el peso de las mismas durante los años 19992010. Sobre la base de la secuencia y el valor de estos indicadores se calcula el índice de competitividad de estos países. Compartimos la idea de que otros índices de competitividad calculados en el mundo son demasiado complicados y no cubren todos los países. En este trabajo calculamos un índice de competitividad y tratamos de demostrar que es posible encontrar otros métodos más sencillos para hacerlo.

\begin{abstract}
This paper presents the calculation of a new competiveness index for seventeen countries in Latin America. The position of each country in our index is based on the comparison of eight indicators in four dimensions and the weight thereof throughout the years 1999-2010. The competiveness index of selected Latin-American countries was calculated based on the sequence and value of these indicators, resulting in a ranking of these countries. We hold the idea that other competiveness indices calculated in the world are too complicated and don't cover all the countries. In this paper we presented a competiveness index and we have tried to demonstrate that it is possible to find other, simpler methods to calculate it.
\end{abstract}

Palabras clave: América Latina, competitividad, desarrollo económico, factores institucionales, dimensión económica, dimensión social, tecnología.

Keywords: Latin America, competiveness, economic development, institutional factors, economic dimension, social dimension, technology.

* Lourdes Gabriela Daza Aramayo, de origen boliviano, es maestra en Economía en la Universidad de Economía de Praga, República Checa, donde actualmente funge como directora ejecutiva del Centro de Estudios Latinoamericanos. 


\section{Introducción}

$A_{\text {. }}$ inestables niveles de crecimiento económico. En la actualidad, dicha región se distingue por su constante desarrollo y estabilidad macroeconómica con cambios en la producción, comercio, infraestructura, servicios financieros, educación, instituciones, etc. En los últimos años, esta región ha experimentado un crecimiento significativamente superior a la media económica a pesar de la crisis financiera mundial, gracias a la respuesta oportuna de las políticas económicas de estos países frente a la crisis, junto a los precios altos de materias primas, sobre todo para los productos mineros. Este considerable crecimiento se debe a un aumento significativo de las exportaciones, que también estimulan la acumulación de reservas internacionales, la restauración de la credibilidad de sus economías gracias a la buena gestión de las finanzas públicas, políticas fiscales y monetarias que caracterizan su independencia actual y la autonomía de dichas economías. Todo esto ha contribuido a una posición positiva y alentadora respecto al futuro económico y social de la región. Sin embargo, a pesar de las noticias positivas, al ver un significativo crecimiento económico en América Latina, no debemos olvidar que la región se enfrenta a un gran desafío en lo referente a competitividad, sobre todo al considerar la relevancia e impacto de la globalización y la tecnología en la economía mundial.

\section{La definición de competitividad}

Las primeras definiciones de competitividad por los economistas clásicos Adam Smith y David Ricardo vinculan los factores de producción con el aprovechamiento de las ventajas comparativas. Desde sus orígenes, el término ha estado ligado a la apertura comercial, y desde entonces se ha considerado como la capacidad de un determinado país de insertarse exitosamente en el mercado internacional con el aprovechamiento de sus factores. No obstante, aún cuando el término se ha utilizado continuamente desde hace más de tres siglos, no existe una definición homogénea para precisar el nivel de competitividad.

La dinámica del mercado internacional, actualmente globalizado y caracterizado por la continua inclusión de nuevos actores, la incorporación e importancia de factores de producción como el conocimiento y el desplie- 
gue de los servicios como actividad transferible, han coadyuvado a la falta de una definición común que reproduzca al mismo tiempo tal dinámica, alcances y matices de acuerdo al contexto.

Para algunos autores, la competitividad es un concepto cuya aplicación a las economías nacionales carece de sentido y la obsesión por ella es errónea y peligrosa. Paul Krugman, ganador del Premio Nobel de Economía en 2008 por su análisis de las pautas del comercio y de la localización de la actividad comercial, alerta del peligro que acarrea el buscar la competitividad a cualquier precio. El problema, según Krugman, es que la mayoría de las personas al hablar de la competitividad de una nación lo hacen del mismo modo que al referirse a la competitividad de una empresa privada. Precisamente, Krugman recalca la importancia de no confundir los términos productividad y competitividad.

La definición de esta última se da, generalmente, para un propósito específico. Las definiciones microeconómicas relacionan la competitividad con el éxito de los productos y servicios en los mercados abiertos. De acuerdo con Porter (1990), el éxito en el caso de las economías desarrolladas es determinado principalmente por la capacidad de innovación. Amit (2001), afirma que la competitividad regional está estrechamente vinculada con los avances tecnológicos, la compatibilidad de las tecnologías antiguas con las nuevas y el mantenimiento de las conexiones industriales verticalmente dependientes. La competitividad regional está estrechamente vinculada con la capacidad para adaptarse a las tendencias actuales, el uso de la tecnología de gran alcance y la comercialización de sus conexiones verticales. Malmberg y otros autores et al., (1996) afirman, de igual manera, que la competitividad está asociada con el mantenimiento de sus posiciones en el ámbito regional e internacional y articulada de acuerdo al contexto de la globalización económica. Madies y Prager (2008) mencionan que la competitividad regional está determinada por la capacidad de atraer fuentes internacionales, es decir: inversión, mano de obra calificada, etc.

La competitividad se concibe muy de cerca a la productividad económica y a la teoría de la localización. Desde el punto de vista macroeconómico es mucho más variable (Tvrdoň, Šuranová, 2007) y, a menudo, se asocia con el desarrollo económico sostenible. Además del crecimiento económico, se observa la sostenibilidad social, medioambiental y se considera si el crecimiento económico no se originó a expensas de otras regiones. De hecho, cada región ofrece una ventaja competitiva concreta. En el caso de los países desarrollados, se trata de regiones que ofrecen una mano de obra calificada, un extraordinario sistema de transporte e infraestructura, excelente acceso a los mercados, alta tecnología, buen sistema de salud, 
educación, etc. Por el contrario, las regiones menos desarrolladas ofrecen mano de obra barata y/o sobre todo recursos naturales o materias primas.

De acuerdo con Amit (2001), la competencia regional tiene un carácter convergente, ya que es más probable la introducción de nuevas tecnologías y el aumento de la competitividad en las regiones menos desarrolladas, con bajos salarios y precios de los factores inmóviles de producción. Anderson (2008) menciona que un factor clave para el desarrollo de un entorno económico competitivo es el transporte, no sólo en términos de la accesibilidad, sino también, del contexto de la funcionalidad del mercado de servicios.

A partir de esto, surge una gran interrogante: ¿qué es lo que lleva a una nación a ser más o menos competitiva que otra? Es aquí donde se plantea la mayor discrepancia, pues en función de la perspectiva y el alcance, o del significado mismo que se dé a la competitividad, se tiende a otorgar un valor determinado a ciertas variables o a actores que a otros.

En este trabajo proponemos entender a la competitividad como la capacidad de una región para promocionarse económicamente y a largo plazo frente a otras regiones en un sistema globalizado, respondiendo a las tendencias actuales, a través de la implementación o mejora de tecnología, educación, mano de obra calificada, infraestructura, inversión, seguridad jurídica y sostenibilidad social con la finalidad de alcanzar un desarrollo económico sostenible.

\section{Método para el cálculo del índice}

Con base en esta definición, se elaboró un índice de competitividad basado simplemente en cuatro dimensiones con ocho indicadores. Para ello se tomaron en cuenta las relaciones entre el crecimiento económico y el grado de apertura comercial, inversión extranjera directa, nivel de educación, eficiencia laboral, calidad institucional, nivel de corrupción, desarrollo de la infraestructura y el desarrollo tecnológico, como factores claves para evaluar la competitividad desde un punto de vista macroeconómico. Se utilizó la siguiente fórmula:

$$
\mathrm{CI}_{\mathrm{j}}=\sum_{i=1}^{8}\left(\sum_{t=1}^{12} \frac{\mathrm{I}_{\mathrm{it}}}{\max \left[\mathrm{I}_{\mathrm{ijt}}\right]} * \frac{\frac{0.25 * \mathrm{P}_{\mathrm{k}}}{\mathrm{SDit}}}{\sum_{i=1}^{96}\left(\frac{0.25 * \mathrm{P}_{\mathrm{k}}}{\mathrm{SD}_{\mathrm{it}}}\right)}\right)
$$


El índice de competitividad (IC) compara los indicadores (i) para cada país (j) y año (t) con el valor más favorable entre los otros países (max [Iijt]). El índice comprende cuatro dimensiones, cada una de ellas con dos indicadores. Cada uno de los ocho indicadores de cada país se pondera de manera uniforme (12.5 por ciento). Para tomar en cuenta la dinámica de estos factores se dividió uniformemente en tres períodos de cuatro años: 1999-2002, 2003-2006 y 2007-2010. El ajuste final para la proporción de cada indicador se hace sobre su desviación estándar y se vuelven a calcular de nuevo a 100 por ciento (suma de la desviación estándar total). Se les da un menor peso a los indicadores con mayor desviación estándar y la variabilidad como el PIB. Por otro lado, se les da mayor peso a los indicadores con menor desviación estándar y a la variabilidad como la inversión extranjera directa. El peso uniforme total es de 12.5 por ciento para cada indicador. ${ }^{1}$

\section{Datos}

Se seleccionaron diecisiete países latinoamericanos, diez de América del Sur, seis de América Central y México. Los datos provienen de fuentes estadísticas internacionales relevantes que trabajan con comparaciones internacionales. La consistencia, coherencia, solidez y comparabilidad de los datos son de gran importancia y provienen de las siguientes instituciones: ${ }^{2}$

- El Banco Mundial (BM WDI).

- La Comisión Económica para América Latina y el Caribe (CEPAL).

- US Oficina del Censo.

- RYCIT y de Heritage Foundation.

- Las oficinas nacionales de estadística de países de América Latina.

\section{Variables}

La renta per cápita, PIB/PBI per cápita: el producto interno bruto per cápita basado en la paridad del poder adquisitivo (PPA) en una unidad artificial

1 Véase Daza Aramayo, Lourdes Gabriela y Marek Vokun, 2012, Measuring Competiveness: Latin-American Experience, International Journal of Trade, Economics and Finance, vol. 3, núm. 6, pp. 398-403.

2 La insuficiencia de datos en el área de migración, tasa de desempleo y datos sobre los gastos en investigación y desarrollo en Guatemala, fue solventada mediante la estimación de los mismos. El método básico de estimación fue OLS, predicción lineal con el binario temporal y espacial. 
del PPP que se mide en precios constantes en dólares internacionales del año 2005. Es un indicador comúnmente utilizado para estimar la riqueza económica de un país. Muchos estudios muestran que la renta per cápita está positivamente correlacionada con la calidad de vida de los habitantes de un país. Se trata de la suma del valor bruto agregado por todos los productores residentes en la economía más cualquier impuesto sobre productos y menos cualquier subsidio no incluido en el valor de los productos. Este indicador de valor agregado fue obtenido gracias a la base de datos del Banco Mundial, una de las bases de datos más completa.

1. La inversión extranjera directa per cápita: esta serie muestra las entradas netas (nuevosflujos deinversiones menos desinversiones) en la economía declarante de los inversores extranjeros dividido entre el número de habitantes. Datos en dólares americanos.

2. La tasa de migración neta: este indicador se mide por cada mil habitantes y se refiere al equilibrio de los emigrantes y los inmigrantes. Los datos provienen de la Oficina del Censo de los EE.UU. Aunque los datos proceden de los censos y se consideran relevantes, algunas series de tiempo son dudosas. En el caso de Paraguay y Brasil, se observó un ritmo constante durante todo el período.

3. La tasa de desempleo: de acuerdo con el método de medición, esta tasa corresponde a la tasa de desempleo real y los datos fueron obtenidos de la CEPAL. El resto de años de los datos que faltaban en la serie de tiempo se calculó utilizando la estimación a través de la variable dependiente con retraso.

4. Los derechos de la propiedad: es una evaluación que realiza la Heritage Foundation de la capacidad de las personas para acumular propiedad privada, garantizada por un marco legal claro. Este índice mide el grado en que las leyes del país protegen los derechos de propiedad privada y la medida en que su gobierno hace cumplir dichas leyes. De igual forma, evalúa la probabilidad de que la propiedad privada sea expropiada y analiza la existencia de corrupción e independencia del poder judicial, así como la capacidad de individuos y empresas para cumplir los contratos.

5. Índices de corrupción: la puntuación de este componente obtenido de la Heritage Foundation se deriva principalmente de la corrupción y del índice de percepción de transparencia internacional (IPC) que mide el nivel de corrupción en ciento 
setenta y ocho países. El IPC se basa en una escala de diez puntos en la que una puntuación de diez indica muy poca corrupción y una puntuación de cero indica un gobierno muy corrupto. Al calificar la libertad frente a la corrupción, el índice convierte los datos en bruto del IPC a una escala del cero al cien.

6. Los gastos de investigación y desarrollo (como porcentaje del PIB): Los gastos en investigación y desarrollo son gastos corrientes y de capital (público y privado) sobre el trabajo creativo realizado de forma sistemática para incrementar el conocimiento, incluido el de humanidades, cultura y sociedad, así como el uso del conocimiento para nuevas aplicaciones. El resultado de I + D abarca la investigación básica, investigación aplicada y el desarrollo experimental. ${ }^{3}$

7. La fuerza laboral con educación superior: es la cuota de los empleados más calificados por sus diferentes logros educativos. Debido a la naturaleza de la investigación en la obtención de datos, existe cierta distorsión. Los datos provienen de la "Elaboración de encuestas en hogares" del Banco Mundial y las oficinas nacionales de estadística. Estas series difieren entre un 5 y 10 por ciento por lo que dimos preferencia a los datos del Banco Mundial.

\section{Resultados}

En las siguientes tablas se observa el Índice de Competitividad obtenido para los diecisiete países de América Latina por zonas geográficas. Para una mejor interpretación se han dividido los resultados en dos zonas, la primera comprende el grupo de países de América del Sur y la segunda, el grupo de países de América Central incluyendo México. En los mismos, se observan las cuatro dimensiones sobre las cuáles ha sido elaborado el índice total, cada una con un 25 por ciento de peso en el índice calculado.

3 Definición del Banco Mundial. 
Tabla 1. Resultados para América del Sur

\begin{tabular}{|c|c|c|c|c|c|}
\hline \multirow{2}{*}{ País } & \multirow{2}{*}{$\begin{array}{c}\text { Indice } \\
\%\end{array}$} & \multicolumn{4}{|c|}{ Dimensión } \\
\hline & & Económica & Social & Institutional & Tecnológica \\
\hline Chile & 83.98 & 26.22 & 20.53 & 25.08 & 12.15 \\
\hline Uruguay & 70.97 & 25.12 & 19.07 & 19.86 & 6.92 \\
\hline Brasil & 64.57 & 19.75 & 19.16 & 13.54 & 12.12 \\
\hline Argentina & 62.34 & 21.50 & 20.39 & 9.94 & 10.51 \\
\hline Colombia & 60.98 & 18.41 & 23.83 & 11.60 & 7.14 \\
\hline Perú & 59.63 & 18.88 & 17.63 & 11.99 & 11.13 \\
\hline Venezuela & 52.68 & 17.98 & 19.79 & 7.61 & 7.30 \\
\hline Ecuador & 52.27 & 16.87 & 18.65 & 8.40 & 8.35 \\
\hline Bolivia & 52.06 & 15.47 & 17.44 & 8.56 & 10.58 \\
\hline Paraguay & 47.46 & 14.89 & 18.79 & 7.84 & 5.94 \\
\hline
\end{tabular}

Fuente: elaboración propia.

A la cabeza de los países de América del Sur se encuentra Chile con el mayor índice de competitividad de acuerdo a los resultados obtenidos mediante esta nueva metodología, seguido por Uruguay, Brasil y Argentina. Los países de América del Sur con el índice más bajo son Paraguay, Bolivia y Ecuador.

Al observar la posición de Chile de acuerdo a este nuevo método, resaltan sobre todo, las dimensiones institucional y tecnológica; en estos rubros, Chile consiguió los porcentajes más altos comparado con los otros países. Dentro de la dimensión económica, podemos observar que este país tiene el segundo PIB per cápita más alto de América Latina, que llega a ser un 68 por ciento más alto que la renta per cápita promedio de la región. También podemos percibir el papel que cumple la inversión extranjera directa per cápita. Es la tercera más alta después de Brasil y Perú y, a nivel per cápita, después de Uruguay y Panamá, y representa una tasa 50 por ciento más alta que el promedio de estos países. ${ }^{4}$

En la dimensión social se analizaron dos variables, la tasa de migración neta y la tasa de desempleo. La tasa de migración en Chile, a diferencia de otros países de la región, exceptuando a Costa Rica, tiene un carácter positivo.

4 Se tomó en cuenta la inversión extranjera directa per cápita para evitar la influencia de la superficie territorial de estos países y enfocarnos a la efectividad de la atracción de recursos. 
El nivel de tecnología también es un factor muy importante en este país al igual que la calidad de la educación, ésta se encuentra entre las más altas en América del Sur después de Argentina. En la dimensión tecnológica se analizan dos indicadores, uno, los gastos de investigación y desarrollo como porcentaje del PIB donde Chile ocupa el segundo lugar después de Brasil, el segundo, la fuerza laboral con educación terciaria, en la que Chile se ubica en una posición intermedia, pues se encuentra entre Perú, Bolivia, Panamá y Costa Rica con 37.1 por ciento.

Chile es conocido a nivel mundial como una de las economías más fuertes de América Latina, posee una economía abierta con diferentes acuerdos comerciales con más de cincuenta y ocho países, donde funciona el modelo neoliberal desde 1973 y donde la dimensión institucional se caracteriza justamente por su buen funcionamiento. Esto atrae la inversión extranjera directa gracias a la seguridad institucional, el respeto a la propiedad privada y a un relativo bajo nivel de corrupción, la cual se refleja en la dimensión institucional, misma que ha sido analizada con base en los datos de la Heritage Foundation. De acuerdo a éstos, se puede afirmar que el país ofrece el grado más alto de seguridad de la propiedad privada, ya que cuenta con uno de los sistemas judiciales más eficientes en América Latina junto con un nivel relativamente alto libre de corrupción, 67 por ciento, el más alto en la región.

Hay que mencionar que Chile es considerado uno de los países más accesibles para el intercambio comercial en América Latina, según los datos oficiales del Foro Económico Mundial 2010 que lo posiciona en el puesto número 1. El índice de competitividad coincide con esta fuente de prestigio a nivel internacional. En América del Sur, Chile también ocupa el primer lugar al igual que en este análisis, seguido de Uruguay y Costa Rica. Según los datos oficiales, ocupan el puesto cuadragésimo y cuadragésimo tercero, incluso sobrepasan a Brasil, que se encuentra en el puesto sexagésimo quinto a nivel mundial. Esto podría explicar la colocación de nuestros países de acuerdo a esta metodología nueva que aparentemente tiene resultados similares con un método de cálculo mucho más sencillo que los otros.

En cuanto a Uruguay, estamos hablando de una pequeña economía abierta con una proyección de crecimiento del mercado exterior dirigido especialmente al mercado internacional. Tiene el nivel más alto de banda ancha en América Latina. Uruguay es miembro del Mercosur junto con Argentina, Brasil y Paraguay, una zona de libre comercio con un PIB de más de dos mil millones de dólares. El crecimiento económico de este país se encuentra después de Argentina, Chile y México; sin embargo, la inver- 
sión extranjera directa se encuentra por debajo del promedio en la región aún cuando el nivel per cápita es el más alto. La dimensión institucional es uno de los aspectos fuertes de este país, en lo que se refiere al respeto a la propiedad privada se encuentra después de Chile y, de acuerdo a los datos del 2010, tiene el mismo nivel libre de corrupción, 67 por ciento, el más alto en América Latina. . Además, Uruguay tiene uno de los niveles más altos en escolaridad. Se considera un país seguro junto con Chile y Costa Rica y posee un alto nivel de infraestructura, especialmente en tecnología y agua.

Brasil y Argentina mantienen su posición, como las principales economías de América Latina. Argentina se caracteriza por su alto crecimiento económico, tiene la segunda tasa más alta del PIB en América del Sur, una tasa de desempleo por debajo del promedio en esta región, la cual perdió varios puntos en el índice de respeto a la propiedad privada. Esto podría justificarse con la imposición de algunas medidas proteccionistas del Gobierno y diferentes nacionalizaciones que fueron duramente criticadas por los inversionistas extranjeros. Dicho conflicto representa uno de los problemas actuales junto con las políticas de sustitución de importaciones que el gobierno argentino ha implementado recientemente, lo cual podría convertir a este país sudamericano en uno de los países más proteccionistas. En cuanto a su tasa de migración, es la tercera más alta en Sudamérica por encima del promedio.

Otro de los países que avanzan son Perú y Colombia gracias a los cambios institucionales que han realizado recientemente. Perú ha sorprendido en los últimos años por su estable y gran crecimiento económico. El porcentaje más alto para su ubicación en el índice lo ha conseguido gracias a la dimensión social y económica. Tiene el porcentaje más alto en lo que se refiere a la fuerza laboral con educación terciaria, casi el doble del promedio sudamericano y un porcentaje relativamente alto en lo que se refiere al respeto de la propiedad privada, Perú ha trabajado arduamente en ello los últimos años para atraer a la inversión privada.

Entre los países que ocupan los últimos lugares en Sudamérica se encuentran Venezuela, Paraguay, Bolivia y Ecuador. Bolivia y Paraguay tienen las tasas más bajas de crecimiento económico, Bolivia tiene la tasa de desempleo más alta seguida de Paraguay. Estos cuatro países se caracterizan por su bajo porcentaje en lo que se refiere al respeto de la propiedad privada, poseen tasas de migración por encima del promedio y todos, a excepción de Bolivia, tienen tasas por debajo del promedio en lo que se refiere a la fuerza laboral con educación terciaria. Ecuador ha realizado muchos esfuerzos, este año ha aprobado un nuevo código legal para atraer 
la inversión y aumentar la producción. La reforma da paso, por ejemplo, a incrementos salariales y reducción de impuestos, tratando de crear las condiciones para mejorar la competitividad. Ecuador se encuentra en el puesto centésimo quinto de ciento treinta y nueve economías desde el 2009.

Tabla 2. Resultados para América Central y México

\begin{tabular}{|c|c|c|c|c|c|}
\hline \multirow{2}{*}{ País } & \multirow{2}{*}{$\begin{array}{c}\text { Índice } \\
\%\end{array}$} & \multicolumn{4}{|c|}{ Dimensión } \\
\hline & & Económica & Social & Institutional & Tecnológica \\
\hline Panamá & 69.54 & 27.53 & 19.78 & 11.13 & 11.11 \\
\hline Costa Rica & 68.48 & 23.42 & 17.97 & 15.35 & 11.75 \\
\hline México & 60.03 & 23.60 & 13.87 & 12.90 & 9.67 \\
\hline El Salvador & 48.56 & 17.25 & 11.85 & 13.97 & 5.49 \\
\hline Nicaragua & 46.37 & 15.08 & 17.80 & 8.44 & 5.05 \\
\hline Honduras & 44.47 & 16.20 & 15.16 & 9.15 & 3.96 \\
\hline Guatemala & 41.48 & 15.36 & 12.73 & 9.67 & 3.72 \\
\hline
\end{tabular}

Fuente: Elaboración propia.

A la cabeza de esta región se encuentran Panamá (69.54 por ciento) y Costa Rica (68.48 por ciento) seguidos de México (60.03 por ciento). Panamá debe su posición a su gran crecimiento económico, el segundo en América Central, y a sus grandes avances en infraestructura, tiene siete puertos, un mega aeropuerto, sistema ferroviario y un canal que conecta al océano Pacífico con el Atlántico. Panamá es uno de los países que más ha trabajado por la competitividad en la región. A diferencia de otras regiones, se concentra en los servicios más que en materias primas.

Costa Rica se encuentra entre los pioneros en el área de tecnología y tiene una de las tarifas más bajas en la conexión a internet, también se caracteriza por tener una mano de obra muy calificada. Costa Rica es considerado uno de los países más seguros en América Latina, le siguen Chile y Uruguay. Este país tiene un alto nivel de infraestructura, tiene la tercera tasa de crecimiento económico más alta en América Central por encima del promedio, al igual que la tasa de desempleo más baja. Asimismo, tiene la tasa más alta de fuerza laboral con educación terciaria después de Panamá y el porcentaje más alto de respeto a la propiedad privada.

México debe su posición a su gran crecimiento económico que, incluso después de la crisis, lo ha posicionado como el más alto en esta región, tiene la tercera tasa más alta de respeto de la propiedad privada y la cuar- 
ta más alta de fuerza laboral con educación terciaria. Desde 1980, su economía es liberal y desde entonces, hace fuerte énfasis en la liberalización del comercio. Como resultado, se convirtió en el líder mundial en acuerdos de libre comercio, con acuerdos con más de cuarenta países de todo el mundo. México es uno de los países con mayor cantidad de premios de la UNESCO en Ciencia y ocupa el segundo lugar en términos de publicaciones científicas en América Latina. Cuenta con el sistema educativo de más alta calidad después de Costa Rica y Uruguay, especialmente en los campos técnicos. La mano de obra es disponible y barata. México tiene la segunda mejor calidad en servicios ferroviarios de América Latina, así como buenas carreteras y aeropuertos. La naturaleza anticuada de la legislación laboral mexicana tiende a estimular la economía informal, que es uno de los grandes problemas no solamente de este país sino de toda América Latina. En cuanto a migración, se encuentra entre las más altas de la región. La incertidumbre y la inseguridad son uno de los mayores problemas en México, esto se manifiesta de tres formas: la inseguridad para los residentes mexicanos, el aumento de la delincuencia y el agravamiento del tráfico de drogas.

Los últimos lugares en América Central, de acuerdo al índice desarrollado, le corresponden a Guatemala, Honduras y Nicaragua. Una característica que comparten estos países es el bajo crecimiento económico, muy por debajo del promedio de los países de esta región. Tienen una de las tasas más altas de desempleo, por encima del promedio, es por esto que la dimensión económica en estos países es lo que más les perjudica para mejorar su posición. En cuanto a los indicadores de dimensión social, es necesario destacar el bajo porcentaje de respeto a la propiedad privada, que se encuentra por debajo del promedio al igual que altas tasas de migración.

\section{Conclusiones}

El concepto de competitividad en la economía y la geografía económica es un término relativamente nuevo. En el presente artículo, trabajamos con la siguiente definición de competitividad: "la capacidad de una región a largo plazo para promocionarse económicamente frente a otras regiones, a través de la implementación o mejora de tecnología, infraestructura, aspectos institucionales, educación, cohesión social y desarrollo sostenido".

Para evaluar la competitividad regional de América Latina, se utilizó un índice desarrollado por la Universidad de Economía de Praga, basado en la comparación de los valores de una región determinada (o estado) con el 
valor más favorable. Se evaluó la competitividad de los países de América del Sur, América Central y México, con un total de diecisiete países latinoamericanos. No se incluyeron los países del Caribe debido a la falta de disponibilidad de datos de los mismos. Para evaluar la competitividad regional de estos países se utilizó un índice basado en la comparación de los indicadores macroeconómicos. Hemos demostrado que se pueden utilizar otros métodos más sencillos para calcular el índice de competitividad que pueden responden a la realidad económica actual de una región donde nos enfocamos, sobre todo, a aspectos macroeconómicos y no a indicadores o encuestas directas dirigidas al sector privado. El índice propuesto abarca cuatro dimensiones importantes: económica, social, tecnológica e institucional, donde cada dimensión está compuesta por dos indicadores que consideramos relevantes dentro de la dimensión dada. Los indicadores que hemos utilizado son los siguientes: el producto interno bruto per cápita (PPP), la inversión extranjera directa per cápita, la tasa de migración, la tasa de desempleo, el índice de respeto a la propiedad privada, el índice de corrupción, los datos de investigación y desarrollo como porcentaje del PIB y la fuerza laboral con educación terciaria.

En América del Sur, Chile domina con un índice de 83.98 por ciento, seguido de Uruguay (70.97 por ciento) y Brasil (64.57 por ciento). Por el contrario, de acuerdo a nuestros cálculos y los indicadores analizados, los países menos competitivos de América del Sur resultaron ser Ecuador (52.26 por ciento), Bolivia (52.06 por ciento) y Paraguay (47.46 por ciento). Los países centroamericanos, incluyendo en este grupo a México, que obtuvieron los índices más altos de competitividad en la región de acuerdo a nuestros cálculos fueron Panamá (69.54 por ciento), Costa Rica (68.48 por ciento) y México (49.7 por ciento). Los países que ocupan los puestos más bajos de competitividad en Centroamérica fueron Nicaragua (46.37 por ciento), Honduras (44.47 por ciento) y Guatemala (41.48 por ciento).

La evaluación de estos países debe tomarse con cautela. Con base en este análisis podemos destacar la posición de los diferentes países de América Latina compitiendo de una manera u otra entre países y sistemas que son de alguna manera similares y cercanos, aunado a la división geográfica que se realizó por razones interpretativas. Hemos observado que los países con un bajo índice de competitividad, pese a su sorprendente crecimiento económico, tienen problemas estructurales, ahorran e invierten relativamente poco comparados con otras estructuras del mundo y con los mismos países de la región. Realizan pocas exportaciones y tienen problemas de equidad, todavía comprenden una de las regiones con mayor diferencia entre ricos y pobres. Un tema preocupante es el mal manejo de las mate- 
rias primas, por lo que muchos países están retrocediendo enfocándose solamente a su exportación. Este es un momento clave para diversificar la economía, donde debería haber más tecnología, conocimiento, innovación, valor agregado y mayor nivel de competitividad.

Entre las características comunes que comparten los países con bajos niveles de competitividad se encuentran los factores institucionales y tecnológicos. Los datos parecen indicar que el nivel de respeto a la propiedad privada juega un papel clave en el índice de competitividad. Una posición baja en el índice parece corresponder a los países que no ofrecen seguridad jurídica adecuada a la propiedad privada. Debido a la incertidumbre en estos países, el nivel de inversión extranjera directa se ve afectado negativamente, lo cual tiene un impacto directo en el nivel de la tecnología y la industrialización, así como el desarrollo de infraestructura. Consideramos que ésta es sólo una de las principales barreras para el desarrollo económico y un área clave de mejora de la competitividad de estos países. Pareciera que el aspecto institucional tiene un papel muy importante.

La mayoría de los países de América Latina y el Caribe todavía están rezagados en términos de competitividad en áreas tan importantes como la innovación y la productividad, que es el principal obstáculo para el éxito de la estrategia de crecimiento económico con equidad. Para incrementar su competitividad, los países de América Latina y el Caribe necesitan trabajar en áreas importantes, consideradas como sectores de crecimiento esenciales en las economías desarrolladas, tales como la infraestructura, logística, seguridad, eliminación de las barreras al comercio y la reducción de la asimetría entre la industria, es necesario aprovechar las nuevas oportunidades globales, apoyar las relaciones de negocios de inversión con nuevos socios internacionales, por ejemplo, Asia, el Pacífico y, por supuesto, la Unión Europea. Otro aspecto muy importante es el de la sociedad basada en el conocimiento donde "la innovación tiene una relación directa con otros rubros como el educativo, laboral, difusión de las nuevas tecnologías, competitividad y crecimiento económico y el grado de desigualdad social" (Villavicencio et al., 2012). Esto podría jugar un papel importante en la creación de empleos de calidad, junto con las políticas sociales que podrían mejorar el acceso a los servicios educativos y sociales, mismo que daría lugar a la reducción de las brechas sociales (en ingresos y el empleo de las mujeres) y, por lo tanto, a la promoción de la cohesión social y los derechos humanos unidos al crecimiento de estos países. 


\section{Bibliografía}

Amiti, M., 2001, "Regional Specialization and Technological Leapfrogging", Journal of Regional Science, vol. 41, núm. 1, pp. 149-172.

Anderson, Simon P. y Weslew W. Wilson, 2008, "Spatial Competition, Pricing and Market Power in Transportation: A Dominant Firm Model", Journal of Regional Science, vol. 48, núm. 2, pp. 367-397.

Atkeson, A. y Lee E. Ohanian, 2001, Are Phillips curves useful for forecasting inflation? Quarterly Review, Minneapolis, Research Department of the Federal Reserve Bank of Minneapolis, pp. 2-11.

Cameron, A. C., y Pravin K. Trivedi, 2010, Microeconometrics Using Stata, edición revisada, Texas, Stata Press.

Cordero M., 2005, Comunidad Andina: un estudio de su competividad exportadora,. Comunidad Andina, disponible en http://www.eclac.org/publicaciones/ xml/2/20692/Serie-25.pdf, (consultado el 18 de diciembre de /2012).

Cho, Dong-Sung, 2000, "From Adam Smith to Michael Porter: Evolution of Competitiveness Theory", Asia- Pacific Business Series, vol. 2, World Scientific Pub Co Inc.

Drezner, Z., George O. Wesolowsky y Tammy Drezner, 1998,“On the Logit Approach to Competitive Facility Location”, Journal of Regional Science, vol. 38, núm. 2, pp. 313 - 327.

Libertad económica en el mundo: Informe Anual 2010, 2012, disponible en http:/ / www. elcato.org/libertad-economica-en-el-mundo-informe-anual-2010 (consultado el 18 de diciembre de 2012).

Madies, T. J., y C. Prager, 2008, Innovation et compétitivité des régions, Paris, La documentation Française, disponible en www.ladocumentationfrancaise.fr/rapports-publics/084000589/index.shtml (consultado el 24 de noviembre de 2012).

Malmberg, A. O., I. Solvell y I. Zander, 1996, “Spatial Clustering, Local Accumulation of Knowledge and Firm Competitiveness", en Geografiska Annaler. Series B,Human Geography, vol. 78, núm. 2, Wiley, Swedish Society for Anthropology and Geography, pp. $85-97$. 
Nijkamp, P. y I. Siedschlag, 2011, Innovation, Growth and Competitioness: dynamic regions in a knowledge based economy, Berlin, Springl, pp. 282 -284.

OECD, 2009, Regions at Glance, Paris, OECD.

OECD, 2006, Competitive Cities in the Global Economy, Paris, OECD.

OECD, 2005, Building Competitive Regions: Strategies and Governance, Paris, OECD.

Porter, M., 1990, The Competitive Advantage of Nations, New York, Free Press.

The Global Competitivness Report, 2011-2012, disponible en http://www.incae. edu/es/clacds/informe-competitividad-global-2011-2012.php (consultado el 12 de noviembre de 2012).

Tvrdoň, J. y J. Šuranová, 2007, “Teoretické a praktické otázky vyjadrenia regionalnej konkurenceschopnosti v ekonomike Slovenska" en 2nd Central European Conference in Regional Science, Košice, Technical University of Košice.

Villavicencio, Daniel, Alberto Morales y Marcela Amaro, 2012, “Indicadores y asimetrías sobre la sociedad basada en el conocimiento en América Latina", en Perfiles Latinoamericanos, núm. 40, México, FLACSO.

World Competitiveness Yearbook, (WCY), 2012, disponible en http:/ /www.imd. org/research/publications/wcy/index.cfm, (consultado el 24 de noviembre de 2012).

Recibido el 7 de enero de 2013

Aceptado el 23 enero de 2014 